\title{
(C)LP Tracing and Debugging
}

\author{
Mireille Ducassé \\ IRISA-INSA of Rennes, Campus universitaire de Beaulieu, \\ 35042 Rennes Cedex, France \\ ducasse@irisa.fr
}

Since Ehud Shapiro's "Algorithmic debugging”, in 1983, there has been a continuous, even if not very abundant, flow of work on tracing and debugging for (constraint) logic programming. The tutorial presents trace production techniques, ranging from compiler instrumentations to dedicated meta-interpreters. It reviews work on trace analysis, in particular algorithmic, declarative and rational debugging. It discusses the issue of trace querying and driving. Last but not least, it describes the latest software engineering research on trace mining.

Throughout the presentation, we stress the importance of the nature of the trace data used by the techniques. We show that CLP techniques have inspired a number of work in other communities. We argue that trace mining techniques can easily be applied to CLP. 